\title{
The Preliminary Study on Spatial Correlation of Ocean Sound Field
}

\author{
Jin-bao Weng and Yan-ming Yang \\ Ocean Laboratory of Acoustics and Remote Sensing, Third Institute of Oceanography, State Oceanic Administration \\ No.178, Daxue Road, Siming District \\ Xiamen 361005, Fujian, China \\ +8615750719913,+86-0592-2197605, wengjinbao@tio.org.cn, yangyanming@tio.org.cn
}

\begin{abstract}
The sound field temporal correlation and spatial correlation, which are the foundation of the investigation of underwater signal space-time high-order characteristics, have important value in the underwater acoustic application. The spatial correlation is studied based on the shallow water acoustic propagation experiment data acquired in the northern South China Sea in 2017, and the deep water acoustic propagation experiment data acquired in the western Pacific in 2013. As for the explosive sound signals in shallow water, time domain waveform cross-correlation coefficients between signals from different propagation distance are calculated. In contrast, the linear frequency modulated signals in deep water need additional matched filtering. The signal processing results shows that, the overall spatial correlation is poor and the correlation radius is relatively small in shallow water, the convergence zone has an obviously better spatial correlation than the shadow zone for the deep water situation. The processing result is verified by simulation and analysis.
\end{abstract}

Key words: Spatial Correlation, Ocean Sound Field, Shallow Water and Deep Water

\section{INTRODUCTION}

With the method of computing ocean sound field becoming more and more accurate, the spatial characteristics of sound field, which are very important in the underwater acoustic application, are increasingly concerned. Spatial correlation is consisted of the horizontal transverse correlation, the horizontal longitudinal correlation and the vertical correlation. The horizontal longitudinal correlation depends on the multipath or multimode propagation and the random fluctuation of medium. The vertical correlation depends on the multipath or multimode propagation. In the paper, experimental and numerical research shows some characteristics of the horizontal longitudinal correlation.

This work is supported in part by the National Key Research and Development Program of China under Grant 2016YFC1400103 and Grant 2018YFC1405900, in part by the National Natural Science Foundation of China under Grant 61701129, Grant 41606116, and Grant 61601132, in part by the Scientific Research Foundation of Third Institute of Oceanography, State Oceanic Administration, under Grant 2016016 and Grant 2015008, and in part by the Natural Science Foundation of Fujian Province under Grant 2016J01019. (Corresponding author: Yan-ming Yang)

\section{THEORY}

\section{A. Correlation Coefficient}

Correlation coefficient between two signals can be expressed the maximum absolute value of delay correlation between two signals, and the delay correlation can be expressed as follow:

$$
\operatorname{COR}\left(P_{1}, P_{2}, \tau\right)=\frac{\int_{-\infty}^{\infty} P_{1}(t) P_{2}^{*}(t-\tau) d t}{\sqrt{\int_{-\infty}^{\infty}\left|P_{1}(t)\right|^{2} d t \int_{-\infty}^{\infty}\left|P_{2}(t)\right|^{2} d t}} .
$$

In equation $1, \tau$ represents time delay, $P$ represents sound pressure, $\operatorname{COR}\left(P_{1}, P_{2}, \tau\right)$ represents delay correlation between sound pressure $P_{1}$ and $P_{2}, t$ represents time.

\section{B. Method of Data Processing}

In order to get the correlation coefficient between two signals, the procedures includes two steps as follow.

(1)Determination of time delay

In the first step, the time delay between two signals can determined by take the maximum value of time domain correlation.

(2) Wave form correlation coefficient

In the second step, the two signals are shifted to the maximum agreement according the time delay. Then the wave form correlation coefficient is calculated as the correlation coefficient between the two signals.

\section{EXPERIMENT}

\section{A. Shallow Water Experiment}

A shallow water acoustical experiment was performed in the northern South China Sea in 2017. Explosive charges [100-g charges of trinitrotoluene (TNT)] were used as the sources. The source depth was $50 \mathrm{~m}$ below the sea surface. The bathymetry was almost flat between the source and receiver, the mean water depth was $120 \mathrm{~m}$. The received signals was recorded by a 19-element, 85m-length vertical line array.

Figure 1 and figure 2 show the normalized waveform of the signals recorded by the first and second hydrophones, and the source range recorded by the Global Position System (GPS) was $10.1 \mathrm{~km}$. Figure 3 and figure 4 show the normalized waveform of the signals recorded by the first and second hydrophones, and the source range recorded by the Global Position System (GPS) was $100.2 \mathrm{~km}$. According to the waveforms, all signals in different range had high signal to noise ratio. 


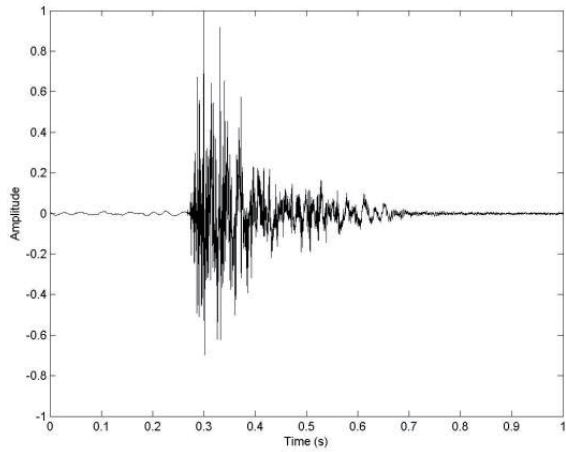

Fig. 1 Normalized waveform of the signal emitted at $10.1 \mathrm{~km}$ range recorded by the first hydrophone.

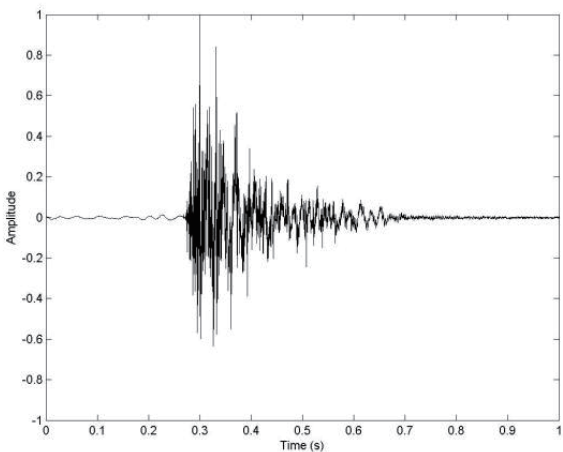

Fig. 2 Normalized waveform of the signal emitted at $10.1 \mathrm{~km}$ range recorded by the second hydrophone.

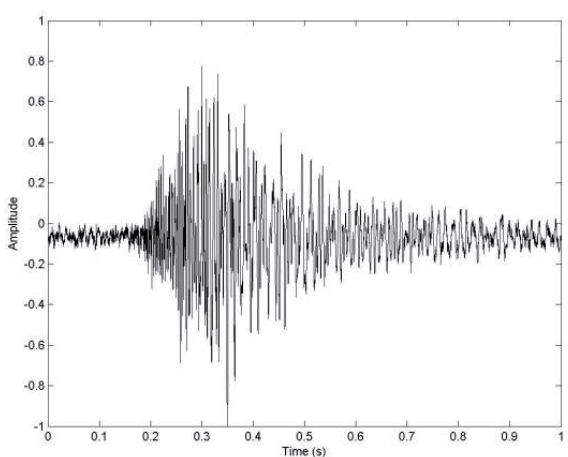

Fig. 3 Normalized waveform of the signal emitted at $100.1 \mathrm{~km}$ range by the first hydrophone.

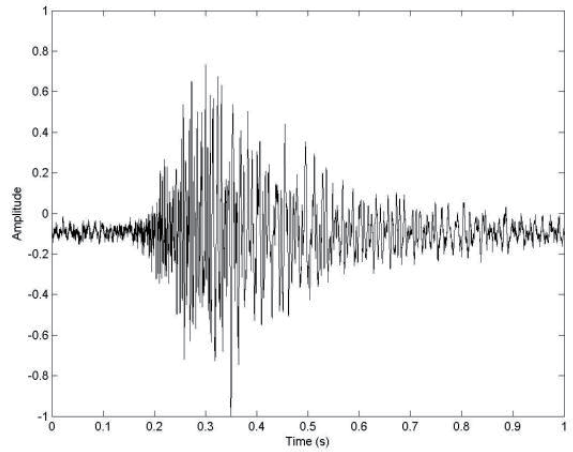

Fig. 4 Normalized waveform of the signal emitted at $100.2 \mathrm{~km}$ range by the second hydrophone.

Figure 5 and figure 6 show the correlation coefficient between the signals from different ranges recorded by the first and second hydrophone. According to the results, the correlation coefficient between received explosive sound signals are relatively poor in shallow water, and the correlation radius is relatively small.

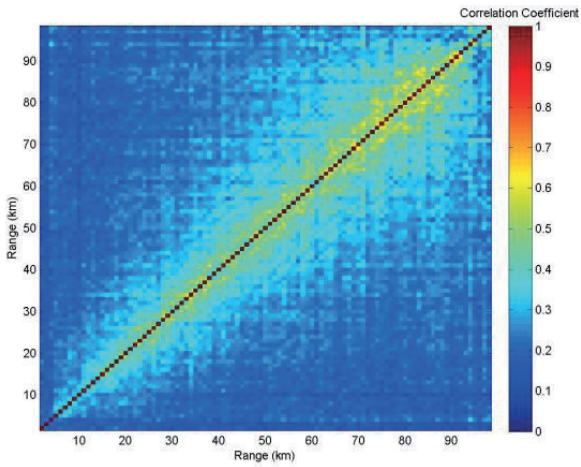

Fig. 5 Correlation coefficient between the signals from different ranges recorded by the first hydrophone.

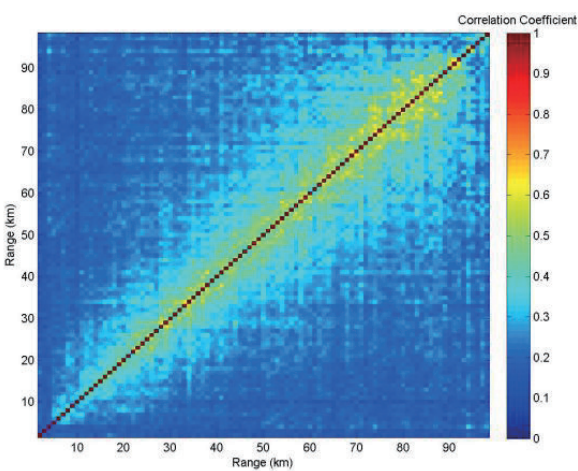

Fig. 6 Correlation coefficient between the signals from different ranges recorded by the second hydrophone.

Figure 7 shows the correlation coefficient of the signals recorded by the first and second hydrophone. According to figure 7 , the correlation coefficient between two hydrophones is very good. 


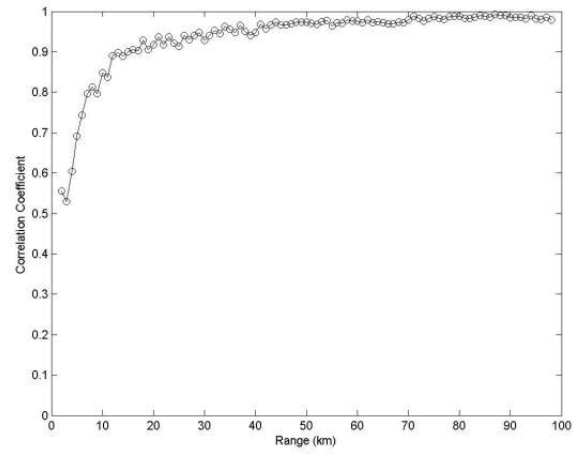

Fig. 7 Correlation coefficient of the signals recorded by the first and second hydrophone.

\section{B. Deep Water Experiment}

A deep water acoustical experiment was performed in the western Pacific in 2013. Towed transducer was used as the sources. Linear frequency modulation (LFM) signal, which's frequency changed from $260 \mathrm{~Hz}$ to $360 \mathrm{~Hz}$ linearly, was emitted once per 160 seconds as the source signal. The source depth was $100 \mathrm{~m}$ below the sea surface. The bathymetry was almost flat between the source and receiver, the mean water depth was $5100 \mathrm{~m}$. The received signals was recorded by a 20 -element vertical line array.

Figure 8, figure 9, figure 10 and figure 11 show the normalized waveform of the signals, and the source range recorded by the Global Position System (GPS) was $2.1 \mathrm{~km}$, $10.3 \mathrm{~km}, 62.2 \mathrm{~km}$ and $70.1 \mathrm{~km}$. According to the waveforms, all signals in different range had high signal to noise ratio.

Figure 12 shows the correlation coefficient between signals from different ranges. According to the results, the convergence zone has an obviously better spatial correlation than the shadow zone for the deep water situation. Besides, there is a strong correlation between the first convergence zone and the second convergence zone.

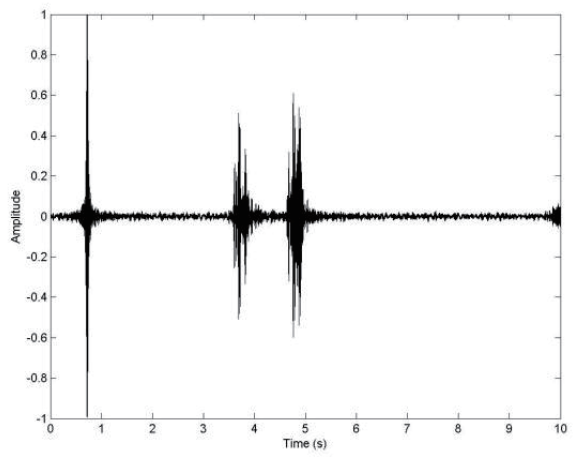

Fig. 8 Normalized waveform of the signal emitted at $2.1 \mathrm{~km}$ range.

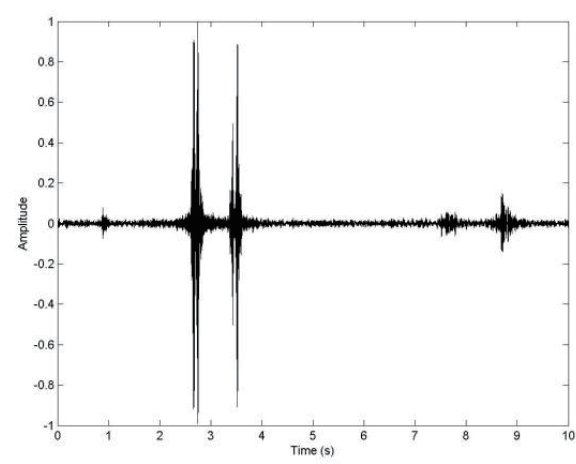

Fig. 9 Normalized waveform of the signal emitted at $10.3 \mathrm{~km}$ range.

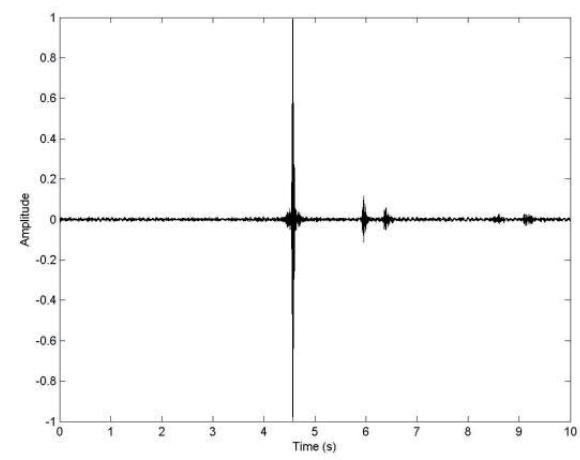

Fig. 10 Normalized waveform of the signal emitted at $62.2 \mathrm{~km}$ range.

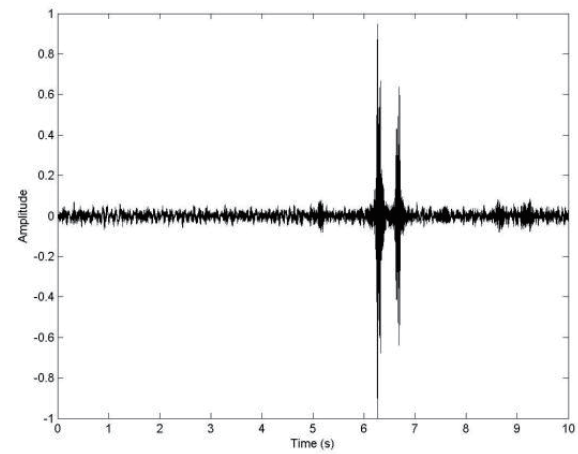

Fig. 11 Normalized waveform of the signal emitted at $70.1 \mathrm{~km}$ range.

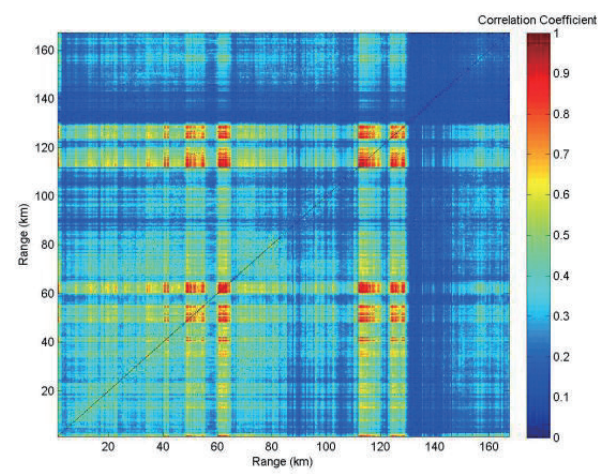

Fig. 12 Correlation coefficient between the signals from different 
ranges.

\section{SIMULATION}

Figure 13 shows the measured sound speed profile. There is a strong negative thermocline between the depths of $20 \mathrm{~m}$ and $120 \mathrm{~m}$, and the channel axis locates at the depth of $1000 \mathrm{~m}$. Figure 14 shows the simulated two-dimensional acoustic transmission loss based on the ray simulation model, and there is a typical deep water sound field distribution with the structure of convergence zones and shadow zones. Figure 15 shows the simulated acoustic transmission loss curve at the depth of $971.4 \mathrm{~m}$, the first convergence zone locates at the range between $50 \mathrm{~km}$ and $70 \mathrm{~km}$, and the second convergence zone locates at the range between $110 \mathrm{~km}$ and $130 \mathrm{~km}$. The locations of convergence zones in figure 15 agree with the locations of high correlation in figure 12. According to the simulation, without the contact with the sea bottom, the signals in convergence zone retain high correlation. However, the signals' correlation diminishes rapidly in shadow zone because of the contacts with the sea bottom.

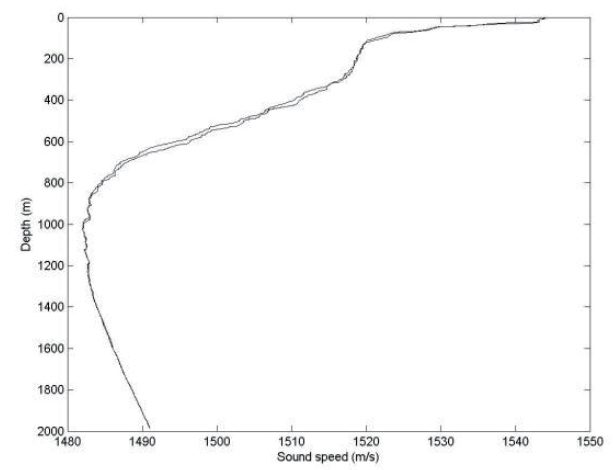

Fig. 13 Measured sound speed profile.

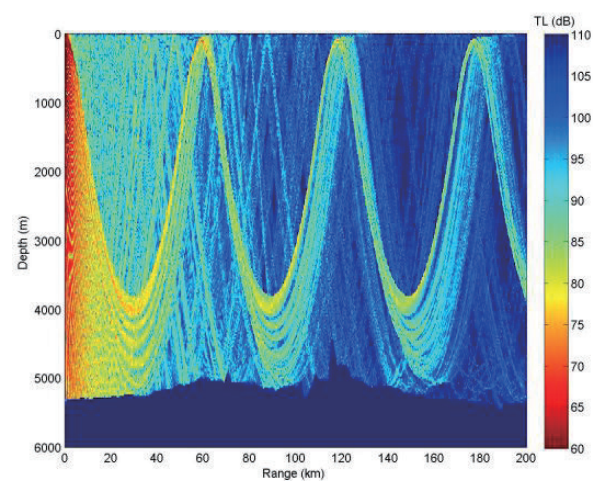

Fig. 14 Simulated two-dimensional acoustic transmission loss.

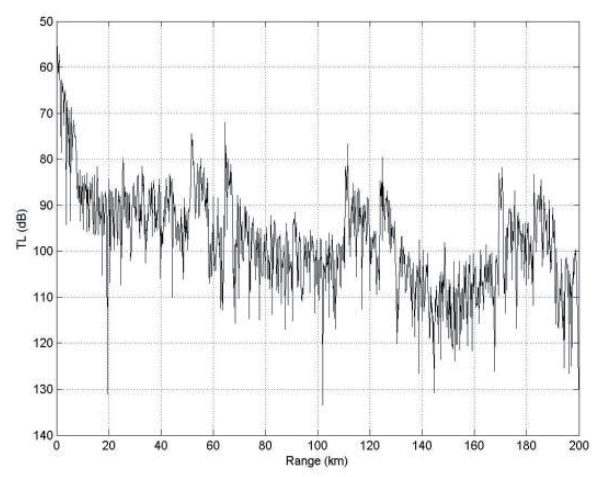

Fig. 15 Simulated acoustic transmission loss curve.

\section{CONCLUSION}

In this paper, experimental research shows that the correlation coefficient of sound field in shallow water and deep water. In the shallow water, the correlation coefficient between received explosive sound signals are relatively poor, and the correlation radius is relatively small. In the deep water, the convergence zone has an obviously better spatial correlation than the shadow zone, and there is a strong correlation among different convergence zones. Based on the comparison between the experiment and the simulation, the spatial correlation diminishes rapidly because of the contacts with the sea bottom, either at shallow water and deep water.

\section{Acknowledgments}

The authors would like to thank all participants of the experiments.

\section{References}

[1] Zaixiao Gong, "The Spatial and Temporal Characteristics of Sound Field with Low Frequency in Shallow Water: Research and Application," unpublished.

[2] Ruichao Zhu and Dinghua Guan, "Spatial horizontal coherence of sound in shallow water", J. Acoust. Soc. Am., Vol. 92(2), Pt.1, August 1992, 956-961.

[3] Qin Wang and Renhe Zhang, "Sound spatial correlation in shallow water", J. Acoust. Soc. Am., 92(2), Pt.1, August 1992, 932-938.

[4] Rozenfeld I., Cable P., Carey W. M., Siegmann W. L., "Spatial coherence in shallow water", OCEANS'97. MTS/IEEE Conference Proceddings, Volume: 1, 1997, pp. 597-601.

[5] A. G. Sazontov and A. L. Mayveyev, "Acoustic coherence in shallow water: theory and observation", Proceedings of the Fifth Europe Conference on Underwater Acoustics, ECUA 2000 .

[6] P. W. Smith, "Spatial coherence in multipath or multimodal channels", J. Acoust. Soc. Am., VOL.60, No.2, August 1976. 